[0212-7199 (2008) 25: 2; pp 81-84] ANALES DE MEDICINA INTERNA Copyright (C) 2008 ARAN EDICIONES, S.L.

AN. MED. INTERNA (Madrid) Vol. 25, N. $^{\circ} 2$, pp. $81-84,2008$

\section{Hemoperitoneo como forma de presentación del carcinoma hepatocelular: experiencia de tres casos con rotura tumoral espontánea y revisión de la literatura}

\author{
M. FERNÁNDEZ-RUIZ, J. M. GUERRA-VALES, J. LLENAS-GARCÍA, \\ J. M. DELGADO-GARCÍA, C. GÓMEZ-PELLICO, A. GONZÁLEZ-BARBER \\ Servicio de Medicina Interna. Hospital Universitario 12 de Octubre. Madrid
}

HEMOPERITONEUM AS PRESENTATION OF HEPATOCELLULAR CARCINOMA: EXPERIENCE IN THREE CASES WITH SPONTANEOUS TUMORAL RUPTURE AND REVIEW OF THE LITERATURE

\begin{abstract}
RESUMEN
El hemoperitoneo secundario a la rotura espontánea de un carcinoma hepatocelular $(\mathrm{CHC})$ supone una complicación potencialmente fatal en ausencia de un abordaje terapéutico apropiado. Constituye una forma de presentación tumoral bien establecida en medios con elevada incidencia de CHC, pero resulta infrecuente en países occidentales, donde se describe en menos del 5\% de los casos. Presentamos tres pacientes atendidos en nuestro centro por hemoperitoneo agudo secundario a la rotura no traumática de un CHC, en los que dicha complicación constituyó la primera manifestación del proceso neoplásico. Realizamos igualmente una revisión de la literatura relacionada con el tema.
\end{abstract}

PALABRAS CLAVE: Carcinoma hepatocelular. Quimioembolización transarterial. Hemoperitoneo. Rotura espontánea.

\begin{abstract}
Hemoperitoneum due to spontaneous rupture of hepatocellular carcinoma (HCC) constitutes a life-threatening situation if no appropriate therapy is provided. This complication is a well-known form of HCC presentation in countries with high incidence of liver tumours, but is an unusual event in Western countries, where it has been described in 5\% or less of cases with HCC. We report three patients admitted to our centre with acute hemoperitoneum secondary to non-traumatic rupture as a first manifestation of not previously diagnosed HCC. A review of the related literature is also performed.
\end{abstract}

KEY WORDS: Hepatocellular carcinoma. Hemoperitoneum. Spontaneous rupture. Transarterial chemoembolization.

Fernández-Ruiz M, Guerra-Vales JM, Llenas-García J, Delgado-García JM, Gómez-Pellico C, González-Barber A. Hemoperitoneo como forma de presentación del carcinoma hepatocelular: experiencia de tres casos con rotura tumoral espontánea y revisión de la literatura. An Med Interna (Madrid) 2008; 25: 81-84.

\section{INTRODUCCIÓN}

El riesgo de rotura tumoral espontánea ha sido bien establecido en las neoplasias primarias del hígado, tanto benignas como malignas (1). El hemoperitoneo asociado a la rotura no traumática de un carcinoma hepatocelular (CHC) supone una forma de presentación relativamente frecuente en medios geográficos con elevada incidencia de enfermedad hepática maligna, como África o el Sudeste Asiático (2-8), en los que la infección por el virus de la hepatitis B (VHB) supone su principal factor etiológico (9); no es raro que esta complicación se describa en sujetos jóvenes sin evidencia previa de hepatopatía (10). Constituye, sin embargo, un evento excepcional en nuestro entorno, donde cerca del $90 \%$ de los casos de CHC asienta sobre hígados afectos de cirrosis establecida $(9,11)$, por lo que su diagnóstico suele realizarse en un contexto clínico diferente (programas de detección precoz, descompensación de hepatopatía crónica de base o dolor abdominal progresivo). Así, la prevalencia de rotura en diversas series occidentales recientes de CHC no supera el 2,8-3,2\% (12-18). No obstante, la elevada tasa de mortalidad, próxima al $50 \%$, que conlleva esta entidad obliga al clínico a garantizar su abordaje temprano y agresivo $(15-17,19)$. Por tanto, consideramos de interés aportar nuestra experiencia en el manejo diagnóstico y terapéutico de esta complicación relativamente infrecuente en la actualidad.

\section{CASOS APORTADOS}

Partiendo de los datos recogidos en el Registro de Tumores del Hospital Universitario 12 de Octubre de Madrid, realizamos un estudio descriptivo retrospectivo de los 236 pacientes con CHC diagnosticados, tratados y sometidos a seguimiento en nuestro centro en el periodo comprendido entre enero de 1999 y diciembre de 2003. Seleccionamos aquellos casos de CHC que debutaron clínicamente en forma de hemorragia intraperitoneal aguda tras la rotura espontánea de su masa tumoral y realizamos la descripción de sus características clínicas, radiológicas y evolutivas, así como de las opciones terapéuticas empleadas en cada caso.

- Caso 1. Varón de 85 años, con historia de hepatopatía crónica en estadio cirrótico secundaria a infección por VHB y varios episodios previos de descompensación ascítico-edematosa y hemorragia digestiva por varices esofágicas. Acude al Servicio de Urgencias por cuadro de dolor abdominal brusco, tipo cólico, acompañado de vómitos, fiebre y desorientación témporo-espacial. A su llegada se objetivó una presión arterial

Trabajo aceptado: 21 de septiembre de 2007

Correspondencia: Mario Fernández-Ruiz. Avda.San Luis, 40, 5C. 28033 Madrid.e-mail: mario_fdezruiz@yahoo.es 
(PA) de 90/50 mmHg, con frecuencia cardíaca (FC) de 73 lpm. Analíticamente destacaba: $\mathrm{Hb}, 10,7 \mathrm{~g} / \mathrm{dl}$; hematocrito, 32,5\%; bilirrubina total, $3,3 \mathrm{mg} / \mathrm{dl}$. La paracentesis diagnóstica demostró hemoperitoneo; la tomografía computadorizada (TC) abdominal, por su parte, informó de la presencia de una gran lesión hepática que ocupaba la práctica totalidad del lóbulo izquierdo, muy sugerente de CHC, acompañada de abundante cantidad de líquido libre perihepático y periesplénico, así como de diversos signos de hipertensión portal (esplenomegalia y varices periesofágicas) (Fig.1). Los niveles séricos de $\alpha$-fetoproteína $(\alpha \mathrm{FP})$ se encontraban por encima del límite superior de la normalidad (61,56 ng/ml; normal, 0 10). Tras desestimar actitud quirúrgica se optó por la realización de un procedimiento de quimioembolización selectiva mediante lipiodol y micropartículas de alcohol-vinilo de la arteria hepática izquierda, obteniendo una hemostasia a corto plazo con resultados radiológicos favorables en controles posteriores. No obstante, el paciente falleció a los tres meses en situación de insuficiencia hepatocelular.

- Caso 2. Varón de 85 años, con antecedentes de diabetes mellitus tipo 2, enfermedad pulmonar obstructiva crónica, fibrilación auri-

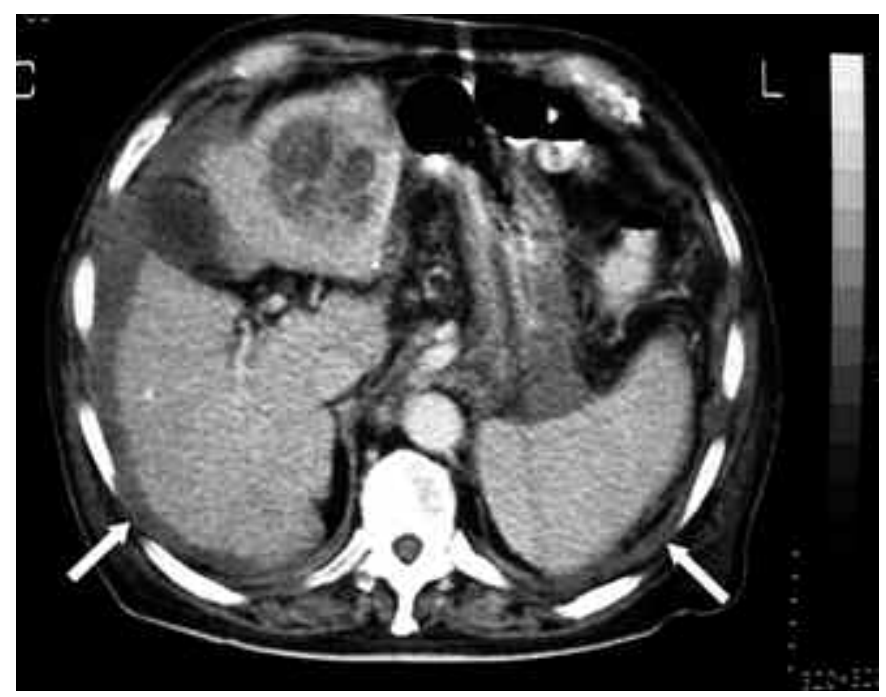

Fig. 1. TC abdominal que muestra una gran lesión polilobulada que ocupa la práctica totalidad del lóbulo hepático izquierdo, sugerente de CHC, acompañada de abundante líquido libre perihepático y periesplénico (flechas).

cular permanente e ictus de origen cardioembólico. No refería historia de hepatopatía crónica ni transfusión de hemoderivados, y negaba el consumo de alcohol. Acude al Servicio de Urgencias por cuadro de una hora de evolución consistente en dolor en piso abdominal superior, de instauración brusca, no acompañado de náuseas ni vómitos. A la exploración presentaba signos de peritonismo difuso, más acusado a nivel de hipocondrio derecho. No desarrolló inestabilidad hemodinámica. Analíticamente destacaba una discreta alteración de las pruebas de función hepática (bilirrubina total, 1,73 mg/dl; GGT, 203 UI/1; fosfatasa alcalina, 746 UI/1), sin anemia ni leucocitosis. La ultrasonografía (US) abdominal urgente informó de la presencia de múltiples lesiones en hígado y bazo, con líquido libre intraperitoneal. El deterioro clínico del paciente, con dolor abdominal y rápida anemización (Hb, 9 g/dl; previa: 10,1), motivó el abordaje quirúrgico mediante laparotomía exploradora, que objetivó la presencia de un hemoperitoneo masivo $(1.000 \mathrm{cc})$ y múltiples nódulos hepáticos de aspecto maligno; el grado de diseminación tumoral desestimó cualquier intervención con intención curativa. El estudio anatomopatológico de la biopsia hepática mostró un CHC bien diferenciado; el nivel de $\alpha$ FP fue de 7,39 ng/ml, con negatividad en las determinaciones serológicas para virus hepatotropos. El paciente falleció a los 12 días de la intervención en el contexto de un fallo multiorgánico.

- Caso 3. Varón de 77 años, con antecedentes de hipertensión arterial y hepatopatía crónica en estadio cirrótico en relación con infección por el virus de la hepatitis C (VHC), con trompocitopenia moderada $(90.000 / \mathrm{ml})$ secundaria a hiperesplenismo. Había sido sometido en varias ocasiones a polipectomías endoscópicas sin evidencia anatomopatológica de malignidad. Acude al Servicio de Urgencias refiriendo clínica de mareo, dolor abdominal y aumento del perímetro abdominal de instauración súbita. Presentaba una PA de 94/60 mmHg y FC de $131 \mathrm{lpm}$, con evidencia analítica de anemización ( $\mathrm{Hb}, 8,6 \mathrm{~g} / \mathrm{dl}$; hematocrito, 23,5\%). Tras la estabilización hemodinámica del paciente, se realizó US abdominal, que demostró abundante líquido libre intraperitoneal con bandas lineales ecogénicas en su interior, así como la presencia de al menos una lesión de gran tamaño en el lóbulo hepático derecho, con halo hipoecoico a su alrededor. La TC abominal urgente confirmó la presencia de esta imagen, adyacente a la cúpula hepática y rodeada por una masa hiperdensa, muy sugerente de $\mathrm{CHC}$ roto a peritoneo con coágulo adherido. Informó además de la existencia de otras dos lesiones; la mayor, de 10 x 11 $\mathrm{cm}$ de diámetro, localizada en el lóbulo hepático izquierdo, presentaba captación muy heterogénea de contraste, congruente con sangrado intratumoral (Fig. 2). Se realizó de forma urgente arteriografía hepática y quimioembolización selectiva de una gran masa hipervascularizada, de aproximadamente $10 \mathrm{~cm}$ de diámetro, que sobresalía del segmento hepático VII, obteniéndose control inmediato de la hemorragia. Los niveles de $\alpha \mathrm{FP}$ alcanzaron rangos virtualmente diagnósticos de CHC (801,7 ng/ml). Tras su ingreso en planta, la situación clínica del paciente experimentó un deterioro progresivo, con fracaso renal y oligoanuria, falleciendo a las tres semanas.

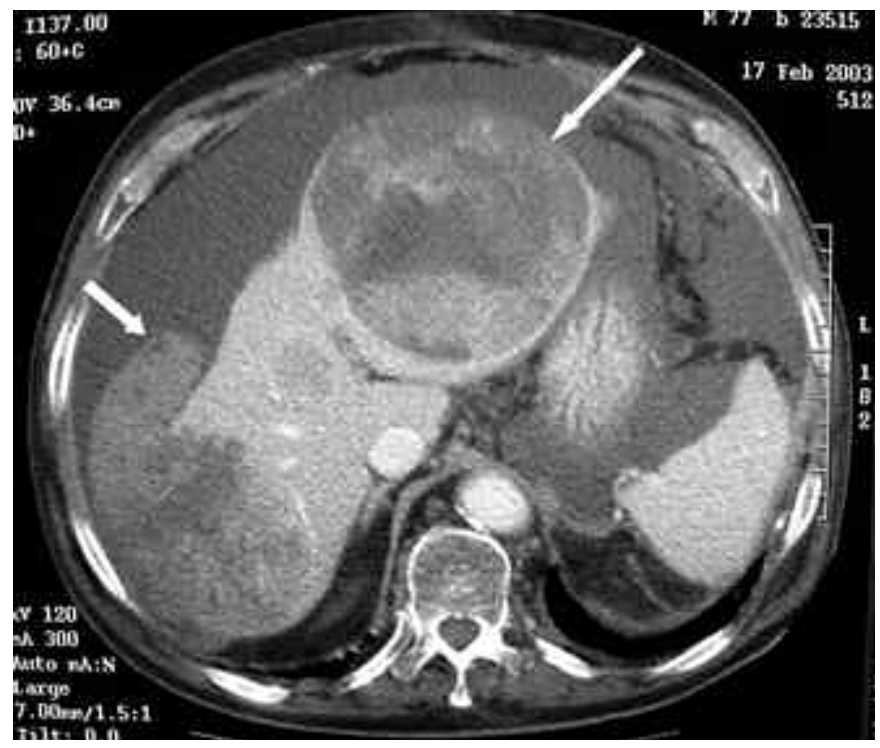

Fig. 2. TC abdominal en el que se observa una lesión de gran tamaño, con captación heterogénea de contraste, en lóbulo hepático izquierdo, sugerente de CHC con sangrado intratumoral (flecha menor), así como una segunda lesión en lóbulo derecho, adyacente a la cápsula hepática, rodeada de una masa hiperdensa en el borde anterolateral del hígado y acompañada de abundante líquido libre intraperitoneal, hallazgos compatibles con la presencia de un CHC roto, coágulo adherido y hemoperitoneo secundario (flecha gruesa).

\section{DISCUSIÓN}

El CHC es la neoplasia primaria maligna más frecuente del hígado (20). Su rotura espontánea constituye una complicación descrita hasta en el 10,5-14,5\% de los casos pertenecientes a series asiáticas de la enfermedad (2-8). Sin embargo, su aparición como primera manifestación tumoral resulta en la actualidad un evento infrecuente en nuestro medio (13-18), más propia de cohortes históricas (12) en las que los programas de segui- 
miento periódico en pacientes cirróticos no se encontraban aún plenamente generalizados. En la tabla I se exponen las diferencias temporales y geográficas en cuanto a la prevalencia de esta entidad a partir de la revisión de varias series de CHC correspondientes a las tres últimas décadas. En nuestro país Castells y cols. (17), sobre una cohorte constituida por 440 pacientes con $\mathrm{CHC}$, comunica la rotura del tumor en tan sólo el 3\% de los casos. Otros autores (13), partiendo de su experiencia con 249 pacientes y un periodo de seguimiento de cinco años, aportan datos similares $(2,8 \%)$. Nuestros resultados arrojan una prevalencia aún menor, con 3 casos de rotura de $\mathrm{CHC}$ en un total de 236 pacientes durante cinco años $(1,3 \%)$. Por el contrario, la rotura espontánea es un evento relativamente común en las neoplasias hepáticas benignas, y supone la primera manifestación del adenoma hepatocelular en cerca del $50 \%$ de las ocasiones $(1,21)$. De forma excepcional se han descrito fenómenos hemorrágicos a partir de localizaciones metastásicas extrahepáticas de un $\mathrm{CHC}$, con sangrado a nivel esplénico, renal, óseo o peritoneal; su rareza y la dificultad de su abordaje justifican el frecuente diagnóstico post mortem $(21,22)$. El mecanismo fisiopatológico íntimo de esta complicación es desconocido, y se han invocado diversos factores: la localización subcapsular del CHC, su tamaño y carácter hipervascularizado, la necrosis espontánea de su masa tumoral, el incremento local de la presión venosa portal secundario a compresión o invasión vascular directa por parte del tumor, o la presencia de hipertensión portal y coagulopatía, propias de órganos cirróticos $(17,19)$. Un reciente análisis retrospectivo de 83 pacientes coreanos con $\mathrm{CHC}$ roto identificó como factores de riesgo asociados de forma independiente a este desenlace la localización periférica de la masa tumoral y una estadificación avanzada en los sistemas de Child-Pugh y Okuda (7). La rotura de un $\mathrm{CHC}$, generalmente espontánea, aunque se ha descrito en ocasiones precipitada tras traumatismos abdominales inaparentes (19), constituye una urgencia vital y obliga a mantener un elevado índice de sospecha clínica en pacientes con cirrosis hepática establecida que presenten dolor y distensión abdominal de instauración súbita acompañadas de rápida anemización e hipotensión, con eventual evolución hacia shock hipovolémico por sangrado masivo en la cavidad peritoneal $(2,10,21)$. Algunos autores, no obstante, sugieren que la inestabilidad hemodinámica no es un fenómeno frecuente; así, Marini et al (1) aporta su experiencia con 20 pacientes que presentaron rotura espontánea de una neoplasia hepática primaria (trece carcinomas y siete adenomas), de los que sólo cuatro (20\% del total) llegaron a desarrollar una situación de shock establecido. Si bien la paracentesis permite confirmar la presencia del hemoperitoneo, la US abdominal y, particularmente, la TC helicoidal constituyen las técnicas de imagen más empleadas en su diagnóstico, en virtud de su accesibilidad y sensibilidad $(15,17)$. El carácter periférico del nódulo tumoral, su protrusión respecto al resto del parénquima hepático, la discontinuidad de su superficie y la presencia de líquido intraperitoneal peritumoral con elevada atenuación radiológica o de un hematoma localizado adyacente a la superficie hepática constituyen hallazgos que deben orientar hacia esta complicación $(10,17,19)$. De manera excepcional puede observarse extravasación del contraste intravenoso en la región perihepática como signo radiológico de sangrado activo (10). No obstante, en numerosas ocasiones sólo se alcanza el diagnóstico de rotura de un $\mathrm{CHC}$ mediante la realización de una laparotomía exploratoria $(8,16)$. El diagnóstico diferencial debe establecerse con otras causas de hemorragia intraperitoneal sin desencadenante traumático, particularmente de naturaleza ginecológica (quistes y carcinoma de ovario) o secundarias a la diseminación peritoneal de un tumor metastático (de origen pancreático, biliar o colorrectal, entre otros) $(15,19)$; también ha sido comunicada esta circunstancia tras la rotura espontánea de grandes varices intra-abdominales o vasos linfáticos dilatados en pacientes cirróticos (23), así como en asociación con otras entidades menos comunes: peliosis hepática, pancreatitis aguda hemorrágica, tuberculosis peritoneal o vasculitis (poliarteritis nodosa) $(19,21)$. El abordaje quirúrgico de la lesión, con resección tumoral y ligadura de la arteria hepática, constituía en décadas anteriores el tratamiento habitual, a expensas de una elevada mortalidad, particularmente en pacientes de edad avanzada o con deterioro severo de la función hepatocelular (3-6,24). La ablación percutánea mediante radiofrecuencia ha sido en ocasiones empleada en tumores de reducido tamaño (menores de $5 \mathrm{~cm}$ ) con resultados satisfactorios (25). No obstante, desde la generalización de las técnicas de embolización intraarterial selectiva, con o sin administración de agentes quimioterápicos, en el manejo del $\mathrm{CHC}$ irresecable, numerosos grupos han propuesto este procedimiento como método terapéutico de elección, inclu-

TABLA I

PREVALENCIA EN DISTINTAS SERIES OCCIDENTALES Y ASIÁTICAS DE ROTURA ESPONTÁNEA CON HEMOPERITONEO AGUDO COMO COMPLICACIÓN ASOCIADA AL CURSO DEL CHC

\begin{tabular}{lccccc}
\hline Autor (referencia) & Año & Procedencia & $\begin{array}{c}\text { Número total de } \\
\text { pacientes con CHC }\end{array}$ & $\begin{array}{c}\text { Número de pacientes con } \\
\text { rotura tumoral espontánea }\end{array}$ & Prevalencia \\
\hline Ong et al (2) & 1972 & Hong Kong & 207 & 30 & 14,5 \\
Chearanai et al (3) & 1983 & $\begin{array}{c}\text { Tailandia } \\
\text { Estados Unidos }\end{array}$ & 508 & 63 & 12,4 \\
Luna et al (12) & 1985 & España & 249 & 5 & 14,3 \\
Calvet et al (13) & 1990 & Bélgica & 35 & 7 & 2,8 \\
Closset et al (14) & 1993 & Italia & 518 & 8 & 2,8 \\
Vivarelli et al (15) & 1995 & Tailandia & 306 & 32 & 10,5 \\
Pawarode et al (4) & 1997 & Italia & 199 & 6 & 3 \\
Vergara et al (16) & 2000 & España & 440 & 14 & 3 \\
Castells et al (17) & 2001 & Taiwán & 535 & 60 & 11,2 \\
Yeh et al (5) & 2002 & Japón & 224 & 83 & 2,7 \\
Mizuno et al (6) & 2004 & Corea & 642 & 17 & 12,9 \\
Min et al (7) & 2004 & Suecia & 530 & 3,2 \\
Kaczynski et al (18) & 2005 & & & & 2 \\
\hline
\end{tabular}


so en presencia de hemoperitoneo masivo o cirrosis en estadio avanzado (17,26-29). Ngan y cols. (28), partiendo de su experiencia con una cohorte de 33 pacientes con rotura espontánea de CHC sometidos a quimioembolización transarterial (QET), comunica la hemostasia del lecho tumoral tras el procedimiento en 32 de ellos $(96,9 \%)$, con una supervivencia mediana global de nueve semanas; la cifra basal de bilirrubina (con un punto de corte de $3 \mathrm{mg} / \mathrm{dl}$ ) se reveló como factor pronóstico independiente. Resultados asimilables en cuanto al control del sangrado a corto plazo son aportados por Castells y cols. (17) en siete pacientes, sin referir ningún caso de deterioro significativo de la función hepatocelular o muerte relacionada con el procedimiento. Otros grupos presentan conclusiones similares en sus respectivas series $(7,27)$. Así, la realización de QET en el manejo del $\mathrm{CHC}$ roto con hemoperitoneo agudo, asociada excepcionalmente a resección quirúrgica (en presencia de una función hepatocelular conservada) (16), parece constituir una técnica relativamente segura, capaz de mejorar a corto plazo la supervivencia, de por sí limitada, de estos pacientes.

En definitiva, presentamos la experiencia acumulada por nuestro centro a lo largo de cinco años en el manejo del hemoperitoneo agudo por rotura espontánea de CHC. La edad, el dete- rioro de la función hepatocelular de base y el estadio tumoral avanzado limitaron las opciones terapéuticas en los tres pacientes aportados. No obstante, y dentro del pronóstico sombrío asociado a esta complicación, con una supervivencia media de 37 días en nuestra serie (rango: 11-81 días), la QET parece constituir el abordaje más efectivo, al permitir un rápido control del sangrado tumoral sin complicaciones asociadas relevantes en los dos casos en los que se realizó. Resulta llamativo que en dos de los tres pacientes comunicados existiera una hepatopatía crónica de base bien documentada, circunstancia a pesar de la cual no eran sometidos a un seguimiento ecográfico periódico que hubiera podido evitar el desarrollo de esta complicación; resulta verosímil suponer que la elevada edad de los pacientes ( 85 y 77 años) condicionara una actitud expectante por parte de sus facultativos. Si bien supone un evento descrito en numerosas series, la incidencia cada vez menor en nuestro medio, lo inespecífico de su clínica y la elevada mortalidad que acompaña a la rotura de un CHC exige mantener una elevada sospecha en su presentación aguda, particularmente en ausencia de hepatopatía crónica conocida, y confirma una vez más la necesidad de aplicar programas de seguimiento periódico mediante US abdominal en individuos afectos de cirrosis hepática.

\section{Bibliografía}

1. Marini P, Vilgrain V, Belghiti J. Management of spontaneous rupture of liver tumours. Dig Surg 2002; 19: 109-13.

2. Ong GB, Taw JL. Spontaneous rupture of hepatocellular carcinoma. Br J Med 1972; 4: 146-9.

3. Chearanai O, Plengvanit U, Asavanich C, Damrongsak D, Sindhvananda K, Boonyapisit S. Spontaneous rupture of primary hepatoma: Report of 63 cases with particular reference to the pathogenesis and rationale treatment by hepatic artery ligation. Cancer 1983; 51: 1532-6.

4. Pawarode A, Voravud N. Ruptured primary hepatocellular carcinoma at Chulalongkorn University Hospital: A retrospective study of 32 cases. J Med Assoc Thai 1997; 80: 706-14.

5. Yeh CN, Lee WC, Jeng LB, Chen MF, Yu MC. Spontaneous tumour rupture and prognosis in patients with hepatocellular carcinoma. Br J Surg 2002; 89: 1125-9.

6. Mizuno S, Yamagiwa K, Ogawa T, Tabata M, Yokoi H, Isaji S, et al. Are the results of surgical treatment of hepatocellular carcinoma poor if the tumor has spontaneously ruptured? Scand J Gastroenterol 2004; 39: $567-70$.

7. Min HJ, Lee OJ, Kang DY, Lee EJ, Lee JH, Kim HJ, et al. The clinical study on spontaneously ruptured hepatocellular carcinoma. Korean J Gastroenterol 2004; 44: 160-7.

8. Hirai K, Kawazoe Y, Yamashita K, Kumagai M, Nagata K, Kawaguchi $\mathrm{S}$, et al. Transcatheter arterial embolization for spontaneous rupture of hepatocellular carcinoma. Am J Gastroenterol 1986; 81: 275-9.

9. Bosch FX, Ribes J, Díaz M, Cléries R. Primary liver cancer: Worldwide incidence and trends. Gastroenterol 2004; 127: S5-S16.

10. Rathod K, Sheth R, Shah P, Rege S. Active contrast extravasation in spontaneous rupture of hepatocellular carcinoma: A rare CT finding. J Postgrad Med 2000; 46: 35-6.

11. Bruix J, Sherman M. Management of hepatocellular carcinoma. Hepatology 2005; 42: 1208-36.

12. Luna G, Florence L, Johansen K. Hepatocellular carcinoma. A 5 year institutional experience. Am J Surg 1985; 149: 591-4.

13. Calvet X, Bruix J, Bru C, Ginés P, Vilana R, Solé M, et al. Natural history of hepatocellular carcinoma in Spain. A five year experience of 249 cases. J Hepatol 1990; 10: 311-7.

14. Closset J, Gelin M, el Nakadi I, Van de Stat J, Lambilliotte JP. Results of surgical resection for hepatocellular carcinoma. Acta Chir Belg 1993; 93: 98-101.

15. Vivarelli M, Cavallari A, Bellusci R, De Raffele E, Nardo B, Gozzetti $\mathrm{G}$, et al. Ruptured hepatocellular carcinoma: An important cause of spontaneous haemoperitoneum in Italy. Eur J Surg 1995; 161: 881-6.

16. Vergara V, Muratore A, Bouzari H, Polastri R, Ferrero A, Galatola G, et

al. Spontaneous rupture of hepatocellular carcinoma: surgical resection and long-term survival. Eur J Surg Oncol 2000; 26: 770-2.

17. Castells L, Moreiras M, Quiroga S, Álvarez-Castells A, Segarra A, Esteban R, et al. Hemoperitoneum as a first manifestation of hepatocellular carcinoma in western patients with liver cirrhosis: Effectiveness of emergency treatment with transcatheter arterial embolization. Dig Dis Sci 2001; 46: 555-62.

18. Kaczynski J, Hansson G, Wallerstedt S. Clinical features in hepatocellular carcinoma and the impact of autopsy on diagnosis. A study of 530 cases from a low-endemicity area. Hepatogastroenterol 2005; 52: 1798-802.

19. Akriviadis EA. Hemoperitoneum in patients with ascites. Am J Gastroenterol 1997; 92: 567-75.

20. Llovet JM, Burroughs A, Bruix J. Hepatocellular carcinoma. Lancet 2003; 362: 1907-17.

21. Polat KY, Akçay MN, Aydinli B, Erdogan F, Kantarci M, Öztürk G, et al. Spontaneous rupture of hepatocellular carcinoma: A case report and review of the literature. Int. J Clin Pract 2005; 59 (Supl. 147): 103-5.

22. Okano J, Shiota G, Horie Y, Mitsuda A, Suou T, Kawasaki H, et al. Rupture of metastasic nodule of the peritoneal surface secondary to hepatocellular carcinoma. Intern Med 1996; 35: 783-4.

23. Léauté F, Frampas E, Mathon G, Leborgne J, Dupas B. Hémopéritoine massif par rupture d'une varice intra-abdominale. J Radiol 2002; 83: 1775-7.

24. Cherqui D, Panis Y, Rotman N, Fagniez PL. Emergency liver resection for spontaneous rupture of hepatocellular carcinoma complicating cirrhosis. Br J Surg 1993; 80: 747-9.

25. Kobayashi M, Ikeda K, Hosaka T, Someya T, Saitoh S, Akuta N, et al. Successful control of ruptured hepatocellular carcinoma with radiofrequency ablation. J Gastroenterol 2004; 39: 192-3.

26. Boyer JC, Zins M, Volgrain V, Sibert A, Najmark D, Vullierme MP, et al. Hemoperitoneum caused by spontaneous rupture of hepatocellular carcinoma. Value and prognostic factors of hepatic embolization. J Radiol 1995; 76: 365-9.

27. Soyer P, Van Beers B, Goffette P, Zeitoun G, Pringot J, Levesque M. Apport de l'embolisation et de la chimio-embolisation au traitement en urgence des hémopéritoines par rupture spontanée de carcinome hépatocellulaire. Gastroenterol Clin Biol 1993; 17: 643-8.

28. Ngan H, Tso WK, Lai CL, Fan ST. The role of hepatic arterial embolization in the treatment of spontaneous rupture of hepatocellular carcinoma. Clin Radiol 1998; 53: 338-41.

29. Trillo P, De Diego J, Niño C, Trillo M, Prego J. Embolización selectiva intraarterial en ruptura espontánea de hepatocarcinoma. Rev Esp Enferm Dig 1996; 88: 569-72. 